\title{
Paul-Peter Tak, Academic Medical Center, University of Amsterdam, The Netherlands (ed): New Therapeutic Targets in Rheumatoid Arthritis. (PIR: Progress in Inflammation Research Series)
}

\author{
2009, 240 p., Hardcover, Birkhäuser Verlag AG, Basel, Switzerland, ISBN 978-3-7643-8237-7
}

\author{
Tracey Toms $\cdot$ Helen Griffiths
}

Published online: 24 March 2010

(C) Springer Basel AG 2010

New Therapeutic Targets in Rheumatoid Arthritis provides an in depth insight into the role of new and evolving drug therapies for the management of rheumatoid arthritis (RA). The book is well designed, with each chapter dedicated to a specific molecular/cellular target fundamental to the underlying pathophysiology of RA. The book first addresses cellular targets ( $\mathrm{B}$ and $\mathrm{T}$ cells), but then systematically examines cytokine targets (e.g. IL-1, IL-6, IL-15, IL-17, IL-18, oncostatin), chemokines and chemokine receptors, intracellular signalling pathways and epigenetic targets. The early chapters focus on several of the newer but existing biologic therapies (e.g. rituximab, anakinara, tocilizumab, abatacept), whereas the later chapters systematically discuss the potential modes of action and available clinical trial data of evolving drug therapies (e.g. certolizumab and JAK3 inhibitors). The data presented in the book are unbiased, providing information on both therapeutic successes as well as therapeutic interventions with a poor clinical outcomes.

The figures/illustrations provide a perfect complement to the text, highlighting the key facts/mechanisms. The only criticism I would have is that some of the figures are in black and white and might have been easier to decipher if they had been presented in colour.

The author contribution to this book is outstanding, with many eminent rheumatological figures contributing knowledge from their specific areas of expertise. Despite the numbers of authors contributing to the book, it reads well and the slight difference in writing styles adds to the overall interest.

The diverse yet simplistic presentation of data in the book will appeal to a large audience including clinical rheumatologists, academic rheumatologists, rheumatology trainees, immunologists and general practitioners.

\footnotetext{
T. Toms NHS Foundation Trust, Russells Hall Hospital,

Dudley, West Midlands DY1 2HQ, UK

H. Griffiths ( $\square)$

Life and Health Sciences, Aston University,

Aston Triangle, Birmingham B4 7ET, UK

e-mail: h.r.griffiths@aston.ac.uk
}

Department of Rheumatology, Dudley Group of Hospitals, 